\title{
Autobiographie, Autofiktion, Metafiktion und Literatur. Der Fall Stadt der Engelvon Christa Wolf
}

\author{
[Autobiography, autofiction, metafiction and literature. The case of Stadt der Enge/by \\ Christa Wolf]
}

\section{Celeste Ribeiro de Sousa ${ }^{1}$}

\begin{abstract}
The key issue of the book Stadt der Engel by Christa Wolf (1929-2011) revolves around the apprehension of reality nowadays. In this work, published in 2010, there is a simple and irrelevant plot which functions, however, as a narrative frame to support all the modes of discourse present in the text: the romanesque, the historical, the autobiographical. There is even a surreal presence of a guardian angel who helps the female protagonist to withstand the vicissitudes of a foreign country and, in particular, to deal with the "discovery" of her own past as informal collaborator of the former GDR secret police (STASI). This essay aims to show how all these discourses are articulated in one literary tissue.
\end{abstract}

Keywords: autobiography; autofiction; metafiction; RDA

Resumo: O tema da obra Stadt der Engel, de Christa Wolf (1929-2011), gira em torno da problemática apreensão do real nos dias de hoje. Há nesta obra, publicada em 2010, uma ação curta e irrelevante que constitui, contudo, uma moldura narrativa a sustentar todos os discursos presentes no texto: o romanesco, o histórico, o autobiográfico. Há até a presença surreal de um anjo protetor que ajuda a protagonista a suportar as vicissitudes de uma estrangeira em terras estranhas e, em particular, a descoberta de seu passado como membro colaborador informal da outrora polícia secreta da DDR (Stasi). Este ensaio objetiva mostrar como todos esses discursos se articulam na forma de um tecido literário.

Palavras-chave: autobiografia; autoficção; metaficção; RDA.

Zusammenfassung: Hauptthema des Buches Stadt der Engel von Christa Wolf (1929-2011) ist die problematische Feststellung der Realität in unseren heutigen Tagen. Dieses Buch, veröffentlicht im Jahr 2010, stellt eine kurze und unbedeutende Handlung dar, welche jedoch als

\footnotetext{
${ }^{1}$ Post-doc in Literaturtheorie und Komparatistik, Universität São Paulo. Sie leitet die Forschungsgruppe RELLIBRA (Linguistische und literarische Beziehungen Brasilien-Deutschland) - www.rellibra.com.br und ist tätig in der Post-Graduierung (deutsche Literatur) an der FFLCH der Universität São Paulo. Email: celeste@usp.br. Dieses Essay wurde in portugiesischer Sprache mit ein paar Veränderungen am 24.04.2012 beim Seminar "GT Literaturas Estrangeiras ANPOLL" an der UNESP - São José do Rio Preto und am 17.10.2013 beim "1. Simpósio de Literatura Alemã: Literatura contemporânea" an der USP vorgelesen.
}

Pandaemonium, São Paulo, v. 17, n. 23, Jun./2014, p. 119-137 
Sousa, Celeste Ribeiro de - Autobiographie, Autofiktion

ein Erzählrahmen funktioniert, der alle Diskurse im Text stützt: den romanhaften, den historischen, den autobiographischen. Sogar die surrealistische Figur eines Schutzengels wird dem Leser geboten, eine Figur, die der Protagonistin als Ausländerin in einem fremden Land hilft, Schicksalsschläge wie die „Entdeckung“ der eigenen Vergangenheit als IM Margarete der Stasi zu überwinden. Absicht dieses Essays ist es zu zeigen, wie alle diese Diskurse im Text zu einem literarischen Gewebe artikuliert werden.

Stichwörter: Autobiographie; Autofiktion; Metafiktion; RDA

\section{Historischer Kontext}

Vor 25 Jahren ist die Berliner Mauer gefallen. Die beiden deutschen Staaten wurden unter der Führung der BRD vereinigt. Die Sowjetunion zerfiel in einzelne Republiken. Der Kommunismus wurde in Frage gestellt. Viele Deutsche wurden glücklich. Andere wurden es nicht. Einige glaubten weiter an die Ideale der Solidarität und Gleichheit der DDR. Manche sahen die Vereinigung als eine große wirtschaftliche Belastung für die BRD. Viele Menschen hatten gehofft und erwartet, dass sich die Wiedervereinigung sehr schnell vollziehen würde, tatsächlich aber war es ein langer - und schwieriger Prozess, der auch heute noch nicht ganz abgeschlossen ist. Echos von diesem schwierigen Prozess sind noch in Christa Wolfs letztem Buch Die Stadt der Engel (2010) zu hören.

Zur Zeit der Veröffentlichung des Buches Stadt der Engel gab es in ganz Deutschland große Erwartungen in Bezug auf die frühere DDR-Schriftstellerin. Vom westlichen Gesichtspunkt aus glaubten und wollten die meisten, dass sie eine vermutliche politische/ethische Schuld in einem Buch erklären würde. Aber sie tat es nicht. Christa Wolf ist am 1. Dezember 2011 gestorben, ohne ein mea culpa in den alten Bundesländern zu machen. Was sie in ihrem letzten Buch Stadt der Engel den Lesern anbietet, ist ein literarisches „Gewebe“, ein poetisches Experiment, in dessen Bau sie versucht, ihre eigene Identität möglichst gut zu verstehen.

Tatsächlich war Christa Wolf der Star der Literatur in der ehemaligen DDR. ${ }^{2}$ Anlässlich ihres Todes bezeichnete sie Der Spiegel als die Schriftstellerin, die die

\footnotetext{
${ }^{2}$ Christa Wolf wurde in der Tat während ihrer literarischen Karriere mit zahlreichen Auszeichnungen geehrt. Die wichtigsten von ihnen sind: der Heinrich-Mann-Preis, 1963; der Theodor-Fontane-Preis des Pandaemonium, São Paulo, v. 17, n. 23, Jun./2014, p. 119-137
} 
Sousa, Celeste Ribeiro de - Autobiographie, Autofiktion

Hoffnungen und Niederlagen einer ganzen Generation erlebt hatte. Daher wurde sie von der Öffentlichkeit sehr geliebt, einer Öffentlichkeit, die sie in ein "literarisches Denkmal" verwandelt hatte, was nur sehr wenigen nach der „Wende“ ${ }^{3}$ gelungen ist.

Einige kurze Bemerkungen über die historische Person Christa Wolf sind nötig, um einen Vergleich zwischen ihr und der namenlosen Protagonistin der Stadt der Engel schließen zu können. Christa Wolf, geborene Ihlenfeld, lebte in der ehemaligen Deutschen Demokratischen Republik und gehörte während vierzig Jahren (von 1949 bis 1989) der SED an, der einzigen Partei im Lande. Sie wurde am 18. März 1929 in Landsberg an der Warthe, heute Polen, geboren. Nach 1945 flohen ihre Eltern nach Mecklenburg-Vorpommern. Von 1949 bis 1953 studierte sie in Jena und Leipzig unter der Leitung von Hans Mayer, einem berühmten Germanisten des Landes. Schon zu dieser Zeit hatte sie sich der Partei angeschlossen. Im März 1959 nahm sie an einem politischen Treffen in Ost-Berlin teil, wo sie als Geheime Informantin (GI) der Stasi (Abkürzung für Staatssicherheit, d.h. für die Geheimpolizei in der DDR), unter dem Decknamen "Margarete" angeworben wurde. Ab dann begann sie dem Regime Informationen über Intellektuelle und Schriftsteller zu geben.

Die Beziehungen zwischen Christa Wolf und der Stasi aber sollten sich bald ändern, als sie sich 1965 bei einem Treffen der Partei gegen die Zensur und gegen die steifen Regeln des sozialistischen Realismus (den in der Sowjet-Union offiziellen

Bezirkes Potsdam, 1972; der Georg-Büchner-Preis, 1980; der Schiller-Gedächtnis-Preis, 1983; der Österreichische Staatspreis für Europäische Literatur, 1985; der Nationalpreis 1. Klasse der DDR, 1987; der Geschwister-Scholl-Preis, auch im Jahre 1987; die Ehrendoktorwürde der Universität Hildesheim, 1990; der Elisabeth-Langgässer-Literaturpreis, 1999; der Nelly-Sachs-Preis, auch im Jahre 1999; der Deutsche Bücherpreis, 2002; der Thomas-Mann-Preis, 2010; der Uwe-Johnson-Preis, auch im Jahr 2010 und die Auszeichnung "Hörkules" im Jahr 2011 für Die Stadt der Engel. Ins brasilianische Portugiesisch wurden zwei der Bücher von Christa Wolf bereits übersetzt: Nachdenken über Christa T., von 1968 (Em busca de Christa T., 1975) und Kassandra, von 1983 (Cassandra, 1990). Ins europäische Portugiesisch wurden auch folgende Texte übertragen: Störfall. Nachrichten eines Tages, von 1987 (Acidente: notícias de um dia, de 1990 ); Medea: Stimmen, von 1996 (Medea: vozes, 1996); Unter den Linden. Drei unwahrscheinliche Geschichten, von 1974 (Unter den Linden - três histórias inverossímeis, 1991).

3 „Wende“: Bezeichnung für die Zeitperiode, die sich vom 9. November 1989, als die Mauer gestürzt wurde, bis zur offiziellen Anerkennung der Wiedervereinigung am 3. Oktober 1990 erstreckt. In der Deutschen Demokratischen Republik wurde „Wende“ im Sinne von Endstation in einer Rede von Egon Kreuz, dem Übergangspräsidenten des Landes, zum ersten Mal am 18. Oktober 1989 benutzt. (http://de.wikipedia.org/wiki/Egon_Krenz). In der Bundesrepublik Deutschland wurde der Begriff zuerst im August 1981 vom Vizekanzler Hans-Dietrich Genscher gebraucht. (http://de.wikipedia.org/wiki/Wende_(Bundesrepublik_Deutschland)).

Pandaemonium, São Paulo, v. 17, n. 23, Jun./2014, p. 119-137 
Sousa, Celeste Ribeiro de - Autobiographie, Autofiktion

Kunststil von 1930 bis $1960^{4}$ ) positionierte. Christa Wolf war also Mitarbeiterin der Stasi und später auch ihr Opfer, weil sie sozusagen eine Art „Dissidentin“ aus Sicht der DDR wurde. Als sie nach einiger Anstrengung das Buch Nachdenken über Christa T. (1968) in einem kleinen Verlag veröffentlichte, wurde sie selbst schon bespitzelt.

1976 wurde der Dichter Wolf Biermann aus der DDR ausgebürgert und Christa Wolf unterschrieb den Protestbrief gegen seine Verbannung, was ihre Marginalisierung durch das Regime noch vergrößerte. Am 3. Oktober 1990 wurde die Wiedervereinigung der beiden deutschen Staaten offiziell besiegelt, aber die Mauer war bereits im Vorjahr, am 9. November 1989, gefallen und die Einrichtung der Gauck-Behörde (StasiUnterlagen-Behörde), d. h. der öffentliche Zugang zu den Akten der Geheimpolizei (zwischen Dezember 1989 und Januar 1990), öffnete auch Christa Wolfs geheimes Leben. Die Offenbarungen verursachten natürlich einen großen Skandal, ähnlich dem, der Günter Grass‘ Bekenntnis zur SS ausgelöst hatte.

Noch 1990 veröffentlichte Christa Wolf Was bleibt, ein autobiografisches ab 1979 geschriebenes Werk, wie die zwei Daten am Ende der Erzählung anzeigen. In diesem Werk beschreibt die Erzählerin das tägliche Ausspioniertwerden einer Schriftstellerin, die sich ganz am Ende des Textes mit der eigenen Protagonistin mit den folgenden Worten identifiziert:

\begin{abstract}
Was sie daran hinderte, in ihrer literarischen und politischen Opposition, ihrer „leisen Dissidenz" gegenüber dem SED-Regime [Sozialistische Einheitspartei Deutschlands] noch weiter zu gehen, weniger Kompromisse zu schließen und die Grenzen des für diesen Machtapparat noch Zumutbaren zu überschreiten, hat sie selbst genau gesehen, beschrieben, analysiert und kritisiert. (WOLF 2007)
\end{abstract}

Das Erscheinen dieses Buches auf dem Markt entfesselte in Deutschland eine breite Debatte über die Mittäterschaft, über die Mitschuld der Schriftsteller in den Regimen, in denen sie leben. Zahlreiche kritische Artikel wurden geschrieben, die die Autorin beschuldigten. Einige jedoch, wie Günter Grass, verteidigten sie. Tatsache aber war die

\footnotetext{
${ }^{4}$ Kurz gesagt, handelt es sich um einen normativen Stil, der dem literarischen Werk die Form eines Verwandlungszeuges der Gesellschaft nach sozialistischer Ideologie sichert. Auf diese Weise müssen alle literarischen Werke fleißige, emanzipierte, exemplarische Figuren darstellen, die immer für die Gemeinde und für die Revolution arbeiten.
} 
Sousa, Celeste Ribeiro de - Autobiographie, Autofiktion

ewige Frage: wie konnte Christa Wolf die Episode (die GI Margarete) vergessen haben? Warum hatte sie niemals darüber gesprochen?

Laut Uwe Wittstocks Rezension ${ }^{5}$ hatte Christa Wolf im Mai 1992 Zugang zu den geheimen Dateien der Stasi über sich selbst, und auch zu denjenigen, die sie selbst als Margarete produziert hatte, was ein Schock für sie war, obwohl der Inhalt der Informationen nur Banalitäten enthielt. Laut Aussagen von Christa Wolf selbst aber waren diese Wochen die schwierigsten ihres Lebens.

Vielleicht hat sie deshalb im September 1992 eine Einladung zu einem neunmonatigen Aufenthalt in das Getty Center in Los Angeles angenommen, wo sie bis Mai 1993 geblieben ist. Sie befindet sich schon in Los Angeles, als sie von ihrer Tätigkeit bei der Stasi schreibt, und den Text in der Berliner Zeitung vom 21. Januar 1993 veröffentlichen lässt.

Am 25. Januar aber macht Der Spiegel bekannt, dass Christa Wolf in der Woche zuvor in der Berliner Zeitung erklärt hatte, dass sie 1959 von der Sicherheitspolizei als Geheime Informantin (GI) und als inoffizielle Mitarbeiterin (IM) angeworben wurde und es bis 1962 blieb. Der Artikel im Spiegel jedoch geht darüber hinaus und enthüllt auch, was die Schriftstellerin weggelassen hatte: dass sie den Decknamen Margarete führte, und dass sie der Geheimpolizei Informationen über Kollegen übergeben hatte.

Noch im Jahr 1993, um Spekulationen über den Gegenstand zu vermindern, lässt Christa Wolf den gesamten Inhalt der Unterlagen über sie unter dem Titel Akteneinsicht Christa Wolf. Zerrspiegel und Dialog. Eine Dokumentation veröffentlichen. Im Jahr 2010 veröffentlicht sie ihr letztes Buch Stadt der Engel oder oder The Overcoat of Dr. Freud im Suhrkamp Verlag.

\footnotetext{
5 "Im Mai 1992 hatte Christa Wolf in der Gauck-Behörde 42 Ordner eingesehen, die von der Stasi angelegt worden waren über die Versuche, sie auszuspionieren - ihre Opfer-Akten. Danach legte ihr die zuständige Mitarbeiterin, entgegen den Regeln der Behörde, einen schmalen Hefter mit Unterlagen vor, in der sie dreißig Jahre zuvor unter dem Decknamen ,Margarete' geführt worden war - ihre Täter-Akte. Eine Verpflichtungserklärung gab es nicht, die Berichte enthielten Banalitäten. [...] Im Januar 1993, also lange nachdem man ihr die alten Spitzelberichte vertraulich vorgelegt hatte, machte sie von Kalifornien aus in einem Zeitungsartikel ihre Stasi-Episode öffentlich.“ (WITTSTOCK 2011)
} 
Sousa, Celeste Ribeiro de - Autobiographie, Autofiktion

\section{Literarischer Kontext}

Stadt der Engel ist eine Erzählung vom Leben der anonymen deutschen Ich-Erzählerin während eines Stipendiums in der Stadt Los Angeles und von ihrer Beteiligung an der Stasi, also eine Art Antwort auf all die Aufregung, die 1993 um die Autorin gewachsen war. Das Buch könnte auch als eine Fortsetzung von Christa Wolfs Kindheitsmuster aus dem Jahr 1976 betrachtet werden, in dem sie durch die Verarbeitung von Erinnerungen an ihre Kindheit und Jugend ihr Leben unter dem Nazi-Regime betrachtet. Hier versucht sie schon, in den komplexen Bildungsprozess ihres eigenen Werdens einzudringen, um das Gedächtnis und seine Verformungen der inneren und äußeren Realität in Frage zu stellen.

Stadt der Engel ist eine Erzählung von 415 Seiten, die mit der folgenden Warnung beginnt:

Alle Figuren in diesem Buch, mit Ausnahme der namentlich angeführten historischen Persönlichkeiten, sind Erfindungen der Erzählerin. Keine ist identisch mit einer lebenden oder toten Person. Ebensowenig decken sich beschriebene Episoden mit tatsächlichen Vorgängen. (WOLF 2010: 6).

Jedoch wird Stadt der Engel, wie bereits erwähnt, durch eine namenlose Protagonistin erzählt, die sich in vielen Punkten des Textes mit der eigenen Schriftstellerin Wolf deckt. Warum hat Christa Wolf diese Warnung geschrieben, die uns unpassend erscheint? Um uns zu erinnern, dass autobiografische Texte unbedingt auch Fiktionen in einem höheren oder niedrigeren Grad sind? Oder um als vehemente literarische Strategie ein letztes Mal zu erklären, dass sie auf die schematischen Regeln des sozialistischen Realismus nicht antwortet?

In der Tat gehört die Erzählung keiner bestimmten Gattung an und in Christa Wolfs eigenen Worten ist sie ein (poetisches) Gewebe, das so gewebt ist, um der Realität möglichst nahezukommen:

Ich wollte ein Gewebe schaffen, das der Wirklichkeit möglichst nahekommt. Ich glaube, es geht fast jedem Schreiber so, dass man unglücklich ist, weil man nicht so schreiben kann, wie man denkt. Was ich in einem Moment erlebe, was in einer Minute. Was ich denke, was ich sehe. Alles auf einmal. Wenn ich schreibe, muss ich eins nach dem anderen aufschreiben, also linear. Man ist sich bewusst: Man kann die Wirklichkeit 
Sousa, Celeste Ribeiro de - Autobiographie, Autofiktion

nicht so fassen, wie sie ist. Um ihr näher zu kommen, stelle ich mir ein Geflecht als Idealform vor. Ich versuche die unterschiedlichsten Erfahrungen, Erlebnisse, Erinnerungen gleichrangig nebeneinanderzustellen, das Ich der Gegenwart, das Du der Erinnerung und dazu noch vieles Mögliche und den Alltag: Also ein in sich verschlungenes Muster, aus dem bestimmte Fäden hervorstreben. (apud KAMMERTÖN \& LEBERT 2010: 3)

Die Erzählung stützt sich auf eine Verflechtung der wichtigsten drei fragmentierten Hauptfäden: 1. das tägliche Leben der Protagonistin im Getty Center in Los Angeles; 2. die Wiedergewinnung der Geschichte der Figur L.; 3. das Schreiben einer sekundären Erzählung, die allmählich und spontan in kleinen Fragmenten entsteht, Fragmenten, die gleich in der Schreibmaschine in großen Buchstaben, die im Text hervortreten, notiert werden. Außerdem gibt es unendliche andere Fragmente von kleineren Fäden, die in die Hauptgeschichte gewebt und verflochten sind. Der Erzählung versuche ich etwas Ordnung zu geben, um davon sprechen zu können.

\section{Alltag am Getty Center in Los Angeles}

In der textuellen poetischen Verwicklung ist der festeste Faden zweifellos der, der das tägliche Leben der Protagonistin in der Stadt LA - genannt Weimar unter Palmen enthält, weil dort die deutschen Exilierten zur Nazizeit, unter ihnen Freud, lebten. In der Pension, wo die Protagonistin in LA wohnt, erscheint auch ein Mantel, der Dr. Freud gehört haben soll. So lässt sich der Untertitel „Oder the overcoat of Dr. Freud“ erklären. Die wörtliche Übersetzung des Stadtnamens ins Deutsche klingt ironisch, und der Zusatz eines zweiten Titels oder Untertitels, der scheinbar und auf den ersten Blick nichts mit dem ersten $\mathrm{zu}$ tun hat, löst alle Grenzen eines möglichen Erwartungshorizontes des Lesers. Alles ist möglich. In der Tat ist LA die Stadt, wo die Protagonistin einen Mantel findet, der eigentlich Dr. Freud gehört hat, wie auf Seite 155 $\mathrm{zu}$ lesen ist.

Der Name "Stadt der Engel" (besonders die Elemente „Engel“, die schützende Wesen par excellence sind) enthält, wie gesagt, eine große Dosis Ironie, denn das unerwartete Elend und die Gewalt, denen die Protagonistin auf den Straßen des reichsten Landes der Welt begegnet, lassen sie ganz erstaunt, stimmlos. Es ist eindeutig 


\section{Sousa, Celeste Ribeiro de - Autobiographie, Autofiktion}

die entschlossene Absicht der Autorin, die negative Seite der Stadt hervorzuheben, um den american way of life zu entmythologisieren.

Der Mantel, der nicht nur die Menschen vor der eisigen Rauheit des Äußeren bewahrt, sondern auch, weil er von Freud ist, vor den Unruhen der inneren Welt schützt, behütet letzten Endes, wenn auch auf eine metaphorische Weise, die Protagonistin vor dem psychologischen Schmerz, den die neuen Entdeckungen um ihren Namen entfesselt haben, denn nicht nur die Autorin sondern auch die Protagonistin hat mit der Stasi verhandelt. Es ist dieser dreifache Schutz - auf der einen Seite der der Engel in der Stadt; auf der anderen Seite der des Mantels in doppeltem Sinne - physisch und psychologisch - , der es erlaubt, dass die Protagonistin ruhiger an ihre Vergangenheit denkt, diese Vergangenheit rekonstruiert, interpretiert, bewertet, während sich die amerikanische Stadt in einen Ort der Enthüllungen verwandelt, aber auch in einen Ort, wo eine Art Epiphanie entsteht.

Das tägliche Leben der Protagonistin im Getty Center in Los Angeles ist ein von in der Stadt erzeugten Mikrogeschichten gewebter Faden, der sich inmitten Erinnerungen der Protagonistin und Aufzeichnungen von historischen Ereignissen abwickelt.

Christa Wolf versucht in diesem Buch, den gesamten subjektiven Prozess ihrer Bildung wiederzugewinnen: a) wie sie Marxistin wurde, b) was dies für sie bedeutete, und c) was verursachte, dass sie sozusagen als eine „Dissidentin“ aus der Sicht der DDR gesehen wurde. Also wichtige autobiographische Aspekte. All dies ist gemischt mit ihren Eindrücken der Landschaft der eigenen Stadt, der Denkmäler, der Wohnungen, wo berühmte deutsche Exilierte damals gelebt hatten - unter ihnen Thomas Mann, Bertolt Brecht, Alfred Döblin, Heinrich Mann und andere -, mit ihren Eindrücken über den american way of life. In diesem Fall beobachtet sie: die Spannung zwischen Reichen und Armen; die Fast-Food- und Konsumgesellschaft; die Begegnung mit dem Buch der buddhistischen Nonne Pema Chödrön The wisdom of no escape, das ihr eine Freundin aus LA geschenkt hat, und das sie liest; die Begegnung mit den Schwarzen; die Almosen; die Nachrichten über Spiritismus, über UFOs, über eine indianische Gemeinschaft, die sie besucht. Auch die politischen Diskussionen zwischen ihr und den anderen Gästen im Center z. B. über die Vor- und Nachteile des Kapitalismus und des Sozialismus. Überrascht wird sie auch von der Unverständlichkeit der Leute in 


\section{Sousa, Celeste Ribeiro de - Autobiographie, Autofiktion}

Amerika, denn sie glauben ohne den Schatten eines Zweifels, dass ihre Lebensweise die beste für die ganze Welt ist, d.h. man sollte nur für den Gewinn und Erfolg leben. Ironische Befremdung verursacht ihr auch die demokratische Präsidentschaftswahl in den Vereinigten Staaten durch nur 1/3 der Wähler. Mit großer Verwunderung stellt sie auch fest, dass sich die deutschen Einwanderer in den USA noch für ihre Herkunft schämen. Außer den Überraschungen streut Christa Wolf gleichfalls andere Informationen über Amerika, wie zum Beispiel, Rodney Kings Verurteilung, die Wahl von Bill Clinton und George Bush, die Erscheinung des Buchs The secret life of Mr. Hoover von Anthony Summers (1993) und - last but not least - die Verfolgung der Kommunisten.

Schon am Anfang beginnt Christa Wolf das Buch mit einer erstaunlichen Behauptung der Protagonistin: Als sie auf dem Flughafen in Los Angeles ankommt, durch das Zollamt geht und dem Beamten ihren alten Reisepass der Ex-DDR zeigt (mittlerweile existiert das Land nicht mehr), fragt sie der Beamte: „Germany?“ Da antwortet sie: „Yes. East Germany“, was den Beamten zwingt, seine Vorgesetzten zu fragen, die aber keine Probleme schaffen. Der Beamte jedoch widersteht der Neugier nicht und fragt sie noch einmal: „Are you sure this country does exist?“ Ohne zu zögern, antwort sie wieder: „Yes, I AM.” Doch in ihrem Inneren wird sie von ihrer spontanen Antwort auch überrascht.

Bei einer anderen Gelegenheit erzählt sie, dass sie während des Mauerfalls im Kino war, und dass sie bei der Rückkehr nach Hause entdeckte, was geschehen war, als sie die vielen Autos in Richtung Berlin-West sah und dabei Angst, Scham, Unterdrückung und Resignation fühlte. Es war passiert. Sie verstand. Der Vergleich mit den hypothetisch imaginierten Gefühlen ihres Vaters, als Hitler zur absoluten Macht griff und ihn verhaftete, gewann Form in ihrem Kopf. Erfahrungen anderer Leute anlässlich der "Wende", die sich von der BRD kolonisiert fühlten, werden in Gesprächen ausgetauscht. Auch Gespräche über den lange verstummten Kontakt mit der Familie. Und wieder kommt ins Licht die Parallele zu den Juden, die 1939 Deutschland verlassen mussten.

Die Verflechtung dieses Erzählfadens entspricht jedoch nicht dem literarischen Gesetz der Spannung und Festigkeit. Er ist wahrhaftig ein dicker und umfangreicher Faden, aber locker, schlaff, ohne eine bestimmte Handlung zu gestalten; er ist aus vielen verschiedenen Rohstoffen gewebt, die sich überlagern, und die in einer schier endlosen Pandaemonium, São Paulo, v. 17, n. 23, Jun./2014, p. 119-137 
Sousa, Celeste Ribeiro de - Autobiographie, Autofiktion

Kette von Ideen-Assoziationen erfolgen; er ist ein Faden, der eine Nachahmung des Gedächtnisprozesses darstellt. Damit versucht die Schriftstellerin, sich der Wahrheit ihrer Wahrheit - maximal zu nähern. Auf diese Weise wechselt der Fokus der Fragmente und Mini-Erzählungen ständig, indem er sich von der Gegenwart hier zu der Gegenwart da, von der Vergangenheit hier zu der Vergangenheit da bewegt. Auf einmal entfaltet sich die Protagonistin sogar in ein Ich und ein $\mathrm{Du}$, welche untereinander in bestimmten Umständen in Dialog treten. Es handelt sich um eine schwierige Archäologie, um eine Sammlung von verstreuten winzigen Fragmenten. Christa Wolf erinnert uns an Hegels Subjektivismus und Dialektik, laut denen die Realität nicht erscheint, wie sie wirklich ist, sondern wie wir sie sehen, d.h. die Realität ist eine ständige Konstruktion des Geistes und wird aus endlosen Perspektiven wahrgenommen.

In dieser Fadenverwirrung gibt es auch ein Fädchen, das die eigenen Betrachtungen der Protagonistin über den Akt des Schreibens in den Text einflechtet: die Grenzen der Wahrnehmung im Kenntniserwerb und die Einschränkungen ihrer Übersetzung aufs Papier. Das Ergebnis ist die Schaffung von etwas Neuem, das trotz der Bemühungen nicht mehr der Vergangenheit entspricht. Die Protagonistin selbst erklärt: die Art und Weise, wie ich die Realität sah und sehe, entspricht nicht der Geschichte.

Im ganzen Buch verwendet die Protagonistin daher jede Gelegenheit, ihr Werden aus ihrer eigenen Vergangenheit auszugraben: die Umstände ihrer Geburt, ihre Ausbildung während des Nationalsozialismus, die Flucht der Eltern in die sowjetisch besetze Zone, die utopischen Ideale des Kommunismus, die sie genährt haben und noch nähren, woran sie geglaubt hat und noch glaubt. Sie spart keine Argumente, ihren Glauben zu verteidigen: Zitate von Goethe, Brecht, Thomas Mann, Adorno, Benjamin, KuBa und anderen, wie dem deutschen Barock-Dichter Paul Fleming (1609 - 1640), in dessen Gedichten die anonyme Protagonistin psychologische Unterstützung sucht. Als Beispiel dieser psychologischen Unterstützung bei Paul Fleming wird in Wolfs Stadt der Engel das Sonett „An sich“ (2010: 156) zitiert. ${ }^{6}$ Von Brecht erwählt Christa Wolf z.

\footnotetext{
6 "Sei dennoch unverzagt, gib dennoch unverloren, /Weich keinem Glücke nicht, steh höher als der Neid,/ Vergnüge dich an dir und acht es für kein Leid, /Hat sich gleich wider dich Glück, Ort und Zeit verschworen.// Was dich betrübt und labt, halt alles für erkoren,/ Nimm dein Verhängnis an, laß alles unbereut/ Tu, was getan muß sein, und eh man dir's gebeut./ Was du noch hoffen kannst, das wird noch stets geboren.//Was klagt, was lobt man doch? Sein Unglück und sein Glücke/ Ist ihm ein jeder selbst. Schau alle Sachen an:/Dies alles ist in dir. Laß deinen eitlen Wahn,// Pandaemonium, São Paulo, v. 17, n. 23, Jun./2014, p. 119-137
} 
Sousa, Celeste Ribeiro de - Autobiographie, Autofiktion

B. das Gedicht „Lob des Kommunismus“, wovon die Zeilen „Die Ausbeuter nennen ihn ein Verbrechen/Aber wir wissen: /Er ist das Ende der Verbrechen" (2010: 82) stammen. ${ }^{7}$ Bei Brecht sucht die anonyme Protagonistin ideologische Unterstützung, politische Legitimität.

Von allen Fädchen aber, die dieser Hauptfaden umfasst, ist das wichtigste Fädchen zweifellos das, welches die Informationen über das Verhandeln der Protagonistin mit der Stasi enthält. Es ist dieses Fädchen, das die Neugier des Lesers erweckt und Erwartungen beim Lesen schafft. Jedoch gibt es keine Aussage, die die Erwartungen befriedigt, so etwas wie Reue, so etwas wie "mea culpa". Obwohl die Protagonistin keine Fundamentalistin, keine Revolutionärin ist, bleibt sie ihrer eigenen, persönlichen sozialistisch/kommunistischen Weltanschauung treu und bedauert, dass diese Weltanschauung nicht vervollständigt wurde.

Die Protagonistin entzieht sich dem Helden-Modell des sozialistischen Realismus: die Wahrheit erscheint ihr nicht als absolut. Außerdem scheint sie ihre Kinder und ihren Ehemann über alles zu lieben. Sie liest paradoxerweise Tarot-Karten, wenn auch nur im Scherz. Sie glaubt an einen Schutzengel, der sie schützt.

Doch die Auseinandersetzung zwischen ihrer persönlichen, subjektiven Weltanschauung und der in der Bundesrepublik markiert die schwierigste, die schmerzvollste Phase ihres Lebens und bringt sie in die psychologische Praxis von Dr. Kim in Los Angeles.

Und eh du förder gehst, so geh in dich zurücke./Wer sein selbst Meister ist und sich beherrschen kann,/Dem ist die weite Welt und alles untertan."

7 "Er ist vernünftig, jeder versteht ihn. Er ist leicht./Du bist doch kein Ausbeuter, du kannst ihn begreifen./Er ist gut für dich, erkundige dich nach ihm./Die Dummköpfe nennen ihn dumm, und die Schmutzigen nennen ihn schmutzig./Er ist gegen den Schmutz und gegen die Dummheit./Die Ausbeuter nennen ihn ein Verbrechen./Aber wir wissen:/Er ist das Ende der Verbrechen./Er ist keine Tollheit, sondern/Das Ende der Tollheit./Er ist nicht das Chaos/Sondern die Ordnung./Er ist das Einfache/Das schwer zu machen ist." 
Sousa, Celeste Ribeiro de - Autobiographie, Autofiktion

\section{Der Wiedergewinn der Geschichte der Figur L.}

L. ist der erste Buchstabe eines Namens, mit dem ein Bündel Briefe an Emma unterschrieben ist. Emma ist eine Freundin der Protagonistin und diese Briefe werden auf eine besondere Weise im Text transkribiert: sie werden durch Anführungszeichen und mit einem größeren linken Rand im Text angekündigt.

Ich ging an das Regal, in dem die Mappe mit den Briefen von L. lag. Ihr zweiter Brief an meine Freundin Emma war vom Januar 1947. Er begann mit Freudenausrufen darüber, daß Emma lebte und daß sie wieder in Verbindung gekommen waren.

„Wenn auch“, schrieb sie weiter, „ein Brief niemals unsere Küchengespräche ersetzen kann, da wirst du mir zustimmen. Weißt Du noch? Wir saßen am Küchentisch, die SBahn fuhr beinahe durch die Stube, Stube und Küche, das war, was Du bezahlen konntest, wir tranken Blümchenkaffee, du warst ja arbeitslos, die Ämter konnten sich eine Suchthelferin nicht mehr leisten, aber ich hielt mich noch als Assistenzärztin in der Armenklinik, in der wir uns kennengelernt hatten. Damals lernte ich auch meinen lieben Herrn kennen. Da wurde mein Leben mir kostbar. Und so ist es geblieben.

So, nun habe ich alte Frau Dir das Wichtigste gesagt, daß ich verrückt bin wie als junges Ding, und ich seh Deinen erstaunt-spöttischen Gesichtsausdruck. Mein Abgesandter, der junge Korrespondent, hat Dir wohl erzählt, daß ich seit langem als Psychoanalytikerin arbeite.

Und, da ich Deine Neugier kenne: Ja. Auch seine Frau, Dora ist noch da, sie leben zusammen wie eh und je. Lach nicht. Nein zum Lachen ist es nicht.

Während ich dies schreibe, steigt alles wieder in mir auf. Ich seh Dich. Weißt Du eigentlich, wie schön Du damals warst?"“

War Emma schön? Nicht, als ich sie kannte. Da hatte sie ihre Haftzeit im Gefängnis in dem mecklenburgischen Städtchen Bützow.... (WOLF 2010: 93-94)

Außer ein paar Habseligkeiten bekommt die Protagonistin nach Emmas Tod dieses Bündel Briefe, ein altes Parteibuch aus den 20er Jahren und Kopien von Emmas Urteilen in den 50er Jahren, als sie zwei Jahre lang in der DDR für unangemessenes Verhalten verhaftet wurde.

Über L. aber weiß die Protagonistin fast nichts. Nur dass sie in den USA lebte und Emmas Freundin oder Begleiterin war. Als die Protagonistin zu diesen Informationen die Erklärung hinzufügt, dass sie glaubt, Emma sei ihre eigene größte, engste Freundin, zeigt sie etwas Eifersucht. 
Sousa, Celeste Ribeiro de - Autobiographie, Autofiktion

Ein Profil für diese Unbekannte zu gestalten und ihr eine schriftliche Form zu geben, verwandelt sich im Buch in das Forschungsobjekt, das die Annahme der Einladung vom Getty Center in Los Angeles rechtfertigt.

Die Daten über diese Geschichte, d.h. L.s Geschichte, erscheinen aber im Text fragmentarisch, auf eine nicht lineare Weise (vgl.: 2007: 19, 21, 56, 62, 64-67, 93-94, 119, 132-133, 165-167, 212, 243-244, 296-298). Schon am Anfang gibt es Schwierigkeiten bei dem Ausgangspunkt der Forschung. L. soll sehr wahrscheinlich der erste Buchstabe eines Eigennamens sein. Jedoch ist er durch das Computerprogramm „Orion“ nicht zu finden. Dann sucht man Informationen über den Namen „Emma“. Im Computer erscheint die folgende Information: Linke Presse in der Weimarer Republik. Es handelt sich um Emma Schulzes 1932 in Frankfurt a. M. herausgegebenes Buch.

Obwohl die Protagonistin dieses Buch schon kannte, hatte es ihr gegenüber ihre Freundin nie erwähnt. Mehrere Seiten vorher geht die Suche in einem Computer der Universitätsbibliothek weiter, jetzt aber unter den folgenden Schlüsselwörtern "Frauen in der USA-Emigration". Zum Schluss entdeckt man, dass Emmas Buch vor kurzem ausgeliehen wurde. Im Speicher kann man aber die folgenden Daten lesen: Emma gehörte zur Kommunistischen Partei. Sehr wahrscheinlich war sie schon zu dieser Zeit mit L. befreundet. Für die Protagonistin bedeutet diese Freundschaft dann eine Möglichkeit, L.s Persönlichkeit durch Emma und die Briefe zu erfassen. Mehrere Seiten weiter präsentiert die Protagonistin bereits ein physisches Profil von L.: mutige Gesichtszüge, nach hinten gekämmte graue Haare, mittlere Körpergestalt, weder dünn noch dick, immer in Bewegung, nüchtern in hochwertiger Kleidung gekleidet (im Gegensatz zu Emma, die das Aussehen verachtete, welches sie für ein Zeichen des Bürgertums hielt).

Die Protagonistin versucht, zwischen den Zeilen der Briefe die Vorsicht, die Selbstlosigkeit, den ständigen Verzicht zu sehen, die L.s Liebe zu einem verheirateten Mann verlangte. Und sie imaginiert die Gespräche zwischen den Liebenden und bemerkt, dass das, was L. über die revolutionären Dogmen ihrer Jugend schreibt, ein Echo in sich selbst findet: es war die Revolution die einzige Möglichkeit, die Menschheit zu retten, nicht die Liebe.

45 Seiten weiter ermöglicht das Briefelesen der Protagonistin-Forscherin, L.s imaginiertes Profil schärfer zu machen: die Gestalt, das Gesicht, die Frisur, die Stimme. 


\section{Sousa, Celeste Ribeiro de - Autobiographie, Autofiktion}

Und 31 Seiten weiter wird ein vorletzter Brief transkribiert. Da entschuldigt sich L. für die lange Pause, denn die Geliebte war gestorben und die Traurigkeit hatte sie überkommen. Diesen Brief erhält Emma schon mit Schilddrüsenkrebs im Krankenhaus. Der letzte Brief hat das Datum vom Mai 1979 und gehört L. nicht mehr, sondern einer Fremden namens Ruth, die Emma L.s Tod kommuniziert. 8 Seiten voraus taucht Ruths Figur auf, diejenige, die Emma den letzten Brief von L. gesandt hatte. Ruth hatte dieses Mal auch der Protagonistin einen weitereren Brief ins Center geschickt. Sie lud die Protagonistin zu einer Diskussion in einer jüdischen Gruppe. Ruth sollte daher, dachte die Protagonistin, nicht nur L. sondern auch Emma kennen.

Die Folge dieser Geschichte erscheint erst 44 Seiten nachher. Auf Seite 296 liest man, dass Ruth tatsächlich eine nichtjüdische Freundin mit dem Namen Lily hatte, die Psychoanalitikerin in Berlin war, die aber während des Nationalsozialismus keinen Raum für ihre Praxis bekommen hatte, und die mit einem jüdischen Liebhaber wohnte. Sie musste also aus Deutschland fliehen. Allerdings wusste die Protagonistin schon von den Briefen alles, was Ruth von Lily erzählt.

\section{Das Schreiben einer sekundären Geschichte}

Inmitten aller zuvor erwähnten erzählerischen Fäden und Fädchen enthüllt ein anderer sehr fragmentierter Erzählfaden eine tiefere nachdenkliche Stimme, die sich im Alleingespräch ausdrückt und die durch die Anwendung von großen Buchstaben sichtbar/hörbar wird. Es ist, als ob diese Stimme unerwartet im Unbewussten oder Unterbewussten gespeicherte Fragen, Probleme (nicht Antworten) zur Bewusstseinsschicht brächte, und sie gleich spontan per Schreibmaschine registrierte, so dass die Naturalität, die Wahrhaftigkeit nicht verloren ginge, so dass sie nicht dem Filter der Vernunft unterworfen würden. Die ersten beiden Sätze dieser ungewöhnlichen Erzählung erscheinen auf Seite 9 und 28 und verdienen einen Kommentar. Es sind: „AUS ALLEN HIMMELN STÜRZEN“ und „VOM ENDE HER ERZÄHLEN“. Die Seite 9 beginnt so: 
Sousa, Celeste Ribeiro de - Autobiographie, Autofiktion

Die wirkliche Konsistenz von gelebtem Leben

Kann kein Schriftsteller wiedergeben.

E. L. Doctorow

\section{AUS ALLEN HIMMELN STÜRZEN}

Das war der Satz, der mir einfiel, als ich in L. A. landete und die Passagiere des Jets dem Piloten mit Beifall dankten, der die Maschine über den Ozean geflogen, von See her die Neue Welt angesteuert, lange über den Lichtern der Riesenstadt gekreist hatte und nun sanft aufgesetzt war. (WOLF 2010: 9)

Mit dem ersten Satz „AUS ALLEN HIMMELN STÜRZEN“ beginnt das Buch gleich nach dem Motto, und findet Parallelen im idiomatischen Ausdruck "aus allen Himmeln fallen", oder "aus allen Wolken fallen". Die Erzählerin ersetzt jedoch das Verb "fallen" durch ein anderes heftigeres: "stürzen", das bedeutet "hinfallen", "heftig fallen". Es gibt also eine direkte Anspielung auf die Landung des Flugzeuges in Los Angeles, aber auch auf andere im Text impliziten Bedeutungsschichten wird in dieser drastischen Bewegung hingewiesen. Das Wort "Himmel", verbunden mit „Engeln“, verweist auf die oberen, unergründlichen, geheimnisvollen Welten, die fast immer mit religiösen Utopien verknüpft sind. Im vorliegenden Fall, in dem die Protagonistin eine Kommunistin ist, könnte die evozierte Utopie die einer perfekten kommunistischen Welt sein, woran sie glaubte. Eine mögliche Deutung könnte ebenfalls zeigen, dass dieselbe Protagonistin im Augenblick der Flugzeuglandung die Erfahrung des Mauerfalls und des Endes der DDR, d.h. der kommunistischen Utopie, auf eine dramatische Weise wiedererlebt. Auch könnte sie durch die Worte ,aus allen Himmeln stürzen“ die Vermutung ausdrücken, dass ihre ganzen Bemühungen umsonst gewesen waren, die Utopie einer klassenlosen Gesellschaft - durch die Unterwerfung unter und das Gehorchen einem totalitären Staat gegenüber - durchzuführen. Außerdem verstärkt die Art und Weise, wie der Satz so emphatisch isoliert im Text erscheint, die noch nicht rationalisierte Perplexität der Protagonistin, denn sie hatte im Grund ihre Privatutopie nicht sterben lassen.

Der zweite Satz „VOM ENDE HER ERZÄHLEN“ weist auf das Ende einer Geschichte hin, auf das Ende der Geschichte der DDR, auf das Ende der eigenen Geschichte der Protagonistin und der Schriftstellerin, denn Stadt der Engel ist ihr letztes Buch (Christa Wolf stirbt ein Jahr später), sowie auch auf die Absicht der Erzählerin, 
Sousa, Celeste Ribeiro de - Autobiographie, Autofiktion

eine Geschichte vom Ende zum Anfang hin aufzubauen. So erscheint der Satz auf Seite 28:

[...] Ja, excellente Reinigung von living room, bedroom, bathroom und kitchen, Mrs. Ascott. Wenn Sie wüßten, wie egal mir das ist.

\section{VOM ENDE HER ERZÄHLEN}

kann auch ein Nachteil sein, man kommt in die Gefahr, sich unwissender zu stellen, als man ist, zum Beispiel, was Mrs. Ascott betrifft, die ich unvermeidlicherweise doch eines Tages treffen mußte, falls das der richtige Ausdruck ist für unsere erste Begegnung. [...] (WOLF 2010: 28)

Also weist dieser zweite Satz auf den Wunsch hin, durch den Entfernungs/Entfremdungsfilter über die Vergangenheit nachzudenken, um zu versuchen, die Entstehung, die Verkettung und die Entfaltung der Überzeugungen zu gestalten, die den Intellekt der Protagonistin, ihre Welt der Emotionen und ihre Welt der Gefühle bilden. In anderen Worten handelt es sich um eine Suche nach der Wahrheit, nach der eigenen Wahrheit der Protagonistin. Es ist ein lobenswerter Versuch, der aber schon am Anfang scheitert, denn so sehr man die Vergangenheit zur Gegenwart bringt, funktioniert der Gedächtnismechanismus auf eine assoziative und selektive Weise, was es unmöglich macht, die Vergangenheit in denselben gleichen Parametern von damals zu gestalten. Dieser Einschränkung ist sich die Protagonistin sehr wohl bewusst, da sie auf Seite 40 in einem anderen Fragment denselben Erzählfaden erklärt:

Und wenn all meine Geschäftigkeit, die verdammt nach Fleiß aussehen soll, nichts weiter wäre als der Versuch, das Tonband in meinem Kopf zum Schweigen zu bringen. Aber ich kann ja noch nicht wissen, welche Untiefe in mir hier umgepflügt oder im Gegenteil zugedeckt werden soll. (WOLF 2010: 40)

Andere Fragmente vertiefen dasselbe Problem. In einem von ihnen ist der Einfluss der Lehre der buddhistischen Nonne Pema Chödrön im Buch The wisdom of no escape offenkundig. Die kommunistische Protagonistin überlegt in Los Angeles:

Die Gelegenheit ist günstig. Warum nicht herausfinden, wie ich wirklich bin, wenn diese Nonne mir ins Gesicht hinein behauptet, dass ich mich durch und durch kenne und doch mit mir befreundet sein könnte. Sie nennt das "Loving kindness" und bringt mich in Verlegenheit, weil ich das nicht ins Deutsche übersetzen kann. Anscheinend haben wir nicht diese Freundlichkeit uns selbst gegenüber. Es gibt Selbsthass und Eigenliebe und Eitelkeit, und auf der anderen Seite der Medaille dieses bohrende Minderwertigkeitsgefühl. Das ist doch merkwürdig. (WOLF 2010: 55) 
Sousa, Celeste Ribeiro de - Autobiographie, Autofiktion

Die Protagonistin fragt sich oft selbst, ob sie eine andere Person wäre, wenn sie unter anderen Umständen erzogen worden wäre. Dies ist eine Frage, die in tiefes Nachdenken führt. Könnte jemand, der innerhalb eines diktatorischen Regimes erzogen wird, in dem die Welt rundum nach eigenen Gesetzen gestaltet wird, die die Verschiedenheit nicht anerkennen, innerhalb eines Regimes, in dem es unmöglich ist zu unterscheiden, was in Wirklichkeit richtig und falsch ist, könnte jemand unter diesen Umständen divergente Ideen in einem solchen begrenzten Universum entwickeln? Und wenn auch jemand divergente Ideen in einem solchen begrenzten Universum entwickeln kann, hat er die Bedingungen, den Preis der Rebellion zu leisten, der immer sehr hoch ist? Hoch ist auch der Preis des Schweigens, wenn die Diktatur fällt, wie im Fall der DDR.

Es sieht so aus, als ob Christa Wolf hinter ihrer Protagonistin durch diese in großen Buchstaben notierten Überlegungen zeigen wollte, dass das Leben nicht einfach linear ist, dass das Leben nicht in exklusive Muster eingesperrt werden kann, die in bestimmten Zeitperioden der Geschichte verlangt werden. Es sind von der Protagonistin die folgenden Worte: "Jede Zeile, die ich jetzt noch schreibe, wird gegen mich verwendet werden." (WOLF 2010: 232).

Und über die Enttäuschungen der Leute sowohl in Europa als auch in den USA in Bezug auf die in diesem Buch geschriebenen Erklärungen der ostdeutschen Schriftstellerin, die mit der Stasi zusammengearbeitet hat, erklärt die Protagonistin:

In der Stadt der Engel wird mir die Haut abgezogen. Sie wollen wissen, was darunter ist, und finden wie bei einem gewöhnlichen Menschen Muskeln Sehnen Knochen Adern Blut Herz Magen Leber Milz. Sie sind enttäuscht, sie hatten auf die Innereien eines Monsters gehofft. (WOLF 2010: 140ff.)

In der Tat ist es schwierig für die Protagonistin, dieses Erzählgewebe, das Verhalten der sogenannten kapitalistischen Welt, zu verstehen, die skrupellos in die Privatsphäre jedes einzelnen Staatsbürgers eindringt. Sie klagt: "Ich möchte sein, wo es noch Geheimnisse gibt. Wo nicht einem jeden jedes Geheimnis mit Gewalt entrissen wird, weil nur so die Welt sauber sein kann.” (WOLF 2010: 381).

Und das Ende dieses Erzählfadens weist auf eine Utopie hin, weist auf die andere Seite des Lebens hin, auf die Bewahrung der Privatsphäre, des Subjektivismus, 
Sousa, Celeste Ribeiro de - Autobiographie, Autofiktion

welche paradoxerweise weder im Kommunismus der Ex-DDR noch im amerikanischen Liberalismus zu finden ist. Eine Utopie, die so angekündigt wird:

Das weiß ich doch schon lange, dass die eigentlichen Verfehlungen diejenigen sind, die im Stillen geschehen, und nicht die öffentlich sichtbaren. Und dass man diese stillen Verfehlungen sehr lange vor sich selbst verleugnet und verschweigt und dass man sie niemals ausspricht. Zäh und dauerhaft hüten wir dieses innerste Geheimnis. (WOLF 2010: 406)

Und dieses andere Gesicht des Lebens wäre "ein wunderbares Welt-Paradies".

Zum Schluss, obwohl die graphischen Differenzierungen zwischen den verschiedenen übergelagerten Reden bleiben und Dr. Freuds Mantel aus der Szene verschwindet, erhalten alle Erzählfäden im großen Erzählgewebe einen Sinn, der Leser versteht ihre Funktion im Ganzen, d.h., dass alle Fäden eine wiedergewonnene Identität gestalten, eine wiedergewonnene Identität, die ins deutsche kollektive Gedächtnis greift. Es handelt sich aber um ein Ganzes, das sich nicht schließt, da das Buch mit diesen folgenden zwei Zeilen endet: "Wohin sind wir unterwegs? Das weiß ich nicht. (WoLF 2010: 415).

\section{Fazit}

Die erste Schlussfolgerung offenbart, dass Christa Wolf die neuesten formalen Innovationen im Bereich der Literatur benutzt, indem sie eine extrem fragmentierte Erzählung mit so zahlreichen Erzählfäden schafft, vielleicht darüber hinaus, was sie je getan hat, eine Erzählung, der die Schriftstellerin einfach den Namen eines narrativen Gewebes gibt, d.h. eines autobiographischen, metafiktionalen und historischen Experiments. Natürlich ist die Technik der Fragmente keine Erfindung im Werk Christa Wolfs; sie wurde besonders in der Romantik benutzt. Auch the stream of consciousness oder der Wechsel bewusst/unbewusst sowie die Montage sind in der Literatur der Moderne und in der Avantgarde zu finden. In diesem Roman von Christa Wolf aber werden diese literarischen Strategien fast $\mathrm{zu}$ einer Abfallgrenze geführt und ausdrücklich gebraucht, um die Komplexität der eigenen Wirklichkeit, der eigenen Identität, der eigenen Wahrheit der Protagonistin/Autorin festzustellen, sie Pandaemonium, São Paulo, v. 17, n. 23, Jun./2014, p. 119-137 
Sousa, Celeste Ribeiro de - Autobiographie, Autofiktion

niederzuschreiben und sie zu verstehen/problematisieren. Das ist eine Tendenz der Literatur in den letzten Jahren. Die zweite Schlussfolgerung, die man nach dem Lesen des Textes erhalten kann, ist, dass die Protagonistin dieses Buches (oder Christa Wolf), obschon sie so unterschiedliche Gegebenheiten erfahren hat, oder genau deshalb, weiterhin an die Utopie einer Welt glaubt, in der sich die Menschen in Fülle vervollkommnen könnten. Die dritte Schlussfolgerung verweist auf die echte Frustration der westlichen Kritik wegen Mangel an größeren und bombastischeren Geständnissen oder Erklärungen über die Intimität der Protagonistin/Schriftstellerin mit der ostdeutschen Geheimpolizei. Dieses Problem wird im Buch auf keine frontale Weise behandelt.

Im Rahmen meiner literarischen Kenntnisse präsentiert das Buch Stadt der Engel - weil es in hohen Grad verwickelt ist - eine textuell etwas originelle literarische Machart und eine Reflexion, die irgendeinen ideologischen Binarismus verneint, indem der Text eine Vielzahl von subjektiven, höchst bedeutungsvollen und imponderablen Daten und Tatsachen literarisch verarbeitet.

\section{Literaturverzeichnis}

BRECHT, Bertolt. Lob des Kommunismus. In: http://erinnerungsort.de/lob-des-kommunismus_124.html. (18/05/2014).

FLEMING, Paul. An sich. In: http://www.text-raum.de/zweiraum/sei-dennoch-unverzagt (18/05/2014).

HAMmeleHLE, Sebastian. Zum Tode Christa Wolfs: Genossin einer ganzen Generation. In: http://www.spiegel.de/kultur/literatur/0,1518,801074,00.html (18/05/2014).

KAMMERTÖN, Bruno \& LEBERT, Stephan. „Was war der Geschmack Ihrer Kindheit, Frau Wolf?" Interview von Christa Wolf. In: Zeit online, 30.06.2010. http://www.zeit.de/2010/27/Christa-Wolf, S. 3 (18/05/2014).

OHNE AUTOR. Die ängstliche Margarete. In: Der Spiegel, 25.01.1993. http://www.spiegel.de/spiegel/print/d-13680284.html (18/05/2014).

WitTSTOCK, Uwe. „Stadt der Engel oder The Overcoat of Dr. Freud“ Christa Wolf denkt in Los Angeles nach über Christa Wolf. In: http://uwewittstock.de/blog/?p=316 (18/05/2014).

Wolf, Christa. Stadt der Engel oder The Overcoat of Dr. Freud. Frankfurt a.M.: Suhrkamp, 2010. . Was bleibt. Frankfurt a. M.: Suhrkamp, 2007.

. Kindheitsmuster. Frankfurt a. M.: Suhrkamp, 2007.

Akteneinsicht Christa Wolf. Zerrspiegel und Dialog. Eine Dokumentation. München: Luchterhand, 1993. 